\title{
OPTIMIZACION DE LA FERTIRRIGACION DE LA FRESA EN LA PROVINCIA DE HUELVA. EFICIENCIA EN EL USO DEL ABONADO
}

\author{
Molina, F. ${ }^{1}$, Gavilán, P. ${ }^{2}$, Ruiz, N. ${ }^{3}$ \\ ${ }^{1}$ Director Departamento Agronómico. Gat Fertiliquidos, Niebla (Huelva). \\ pacomolina@gatfertiliquidos.com \\ ${ }^{2}$ Investigador Titular. IFAPA Centro Alameda del Obispo, Apdo. 3092, 14080 Córdoba, \\ pedrod.gavilan@juntadeandalucia.es \\ ${ }^{3}$ Técnica Especialista Contratada. IFAPA Centro Alameda del Obispo. Apdo. 3092, 14080 \\ Córdoba, natividad.ruiz.baena@juntadeandalucia.es
}

\section{1- Introducción y Objetivos}

En el cultivo protegido de la fresa está muy extendido el control de los parámetros climáticos y de humedad del suelo (Gavilán y col., 2014). Sin embargo, los sistemas para el control de la nutrición han tenido menor grado de desarrollo. El cultivo tiene un periodo crítico que influye en una menor asimilación de nutrientes que coincide con temperaturas bajas del suelo, inferiores a $12^{\circ} \mathrm{C}$, de noviembre a febrero, volumen reducido de raíces al comienzo de la plantación, pocas horas luz, menor actividad fotosintética, plantas forzadas para crecer rápidamente, elevada humedad relativa y baja transpiración.

Además, la fresa, en gran parte de la provincia de Huelva, se cultiva en suelos arenosos, con escaso poder de retención de agua y nutrientes, y con agua de riego que tampoco aporta nutrientes. Se riega con alta frecuencia y, en cierto modo, se puede considerar como un cultivo hidropónico en suelo. Por todo ello, la tendencia en la zona es abonar por concentración de nutrientes equilibrados en el agua de riego.

Los pocos trabajos sobre fertirrigación en fresa en Huelva se han realizado en los comienzos del cultivo y con variedades antiguas (Cadahia, et al. 1988). En cuanto a los estudios sobre las relaciones que existen entre absorción mineral y consumo de agua concluyen que son complejas y reflejan la dificultad para disponer de una estimación fiable de manejo de ambos para aplicarlo posteriormente al manejo de la fertirrigación, debida a la imposibilidad práctica de controlar los parámetros que afectan a un agrosistema en campo. En la práctica, sugieren que ambos procesos no están influenciados con igual intensidad por las condiciones medioambientales. Para niveles de consumo de agua bajos (inferiores a 12 I planta ${ }^{-1}$ mes $^{-1}$ ) existe una clara relación lineal entre la absorción de agua y de nutrientes (Duchein et al., 1994). Sin embargo esta relación no se mantiene para valores elevados de consumo de agua, indicando que la absorción mineral está más negativamente afectada por otros factores como la temperatura del suelo que por la absorción de agua.

En colaboración con el IFAPA, que realiza estudios sobre las necesidades de riego del cultivo, Gat Fertiliquidos, empresa fabricante de fertilizantes líquidos, estudia su fertilización, para poder ayudar a los agricultores a fertirrigar de forma más eficiente.

Al comenzar los ensayos sobre riego se planteaba la duda de, si al bajar el volumen de agua de riego al cultivo manteniendo las concentraciones de fertilizantes que actualmente aplican, al recibir éste menos agua y menos fertilizante, se produciría un déficit de fertilizante, lo que obligaría a aumentar la concentración del mismo en el agua de riego.

Los objetivos de este trabajo fueron: 1) Determinar si cantidades de riego y abono menores a las normalmente usadas afectan a su absorción. 2) Relacionar la eficiencia del abonado con la eficiencia del agua de riego. 


\section{2- Materiales y Métodos}

Los trabajos se realizaron en una parcela comercial del T.M. de Almonte, en la Comarca agrícola Condado-Litoral, cerca de la aldea "El Rocío", en un suelo arenoso, sobre un cultivo de fresa (Fragaria $x$ ananassa) de la variedad Antilla durante la campaña 2013/14. El suelo de la finca del ensayo se califican como arenosos (clasificación USDA), con un 90\% de arena. La empresa propietaria de la finca realizó todas las labores de cultivo propias de la fresa en los ensayos.

Se realizó un ensayo con cuatro tratamientos. Los tres primeros tratamientos (T1, T2, y T3) recibieron volúmenes similares de riego, con el objetivo de cubrir las necesidades de agua del cultivo. La diferencia entre ellos estuvo en el caudal de la cinta de riego usada, cintas de 2.5, 3.8, y $5 \mathrm{I} \mathrm{h}^{-1} \mathrm{~m}^{-1}$ para T1, T2, y T3, respectivamente. T4 recibió diferente volumen de riego respecto a los anteriores, basados en el volumen real utilizado en la finca, para comparar nutrición mineral de la planta con riego menor a lo utilizado en la finca por los agricultores de la zona. Todos los tratamientos se regaron con la misma concentración de nutrientes $(\mathrm{CE})$ en agua, pero con diferentes volúmenes de riego. Más detalles sobre el experimento pueden encontrarse en Gavilán y col. (2014)..

Se realizó un seguimiento analítico mensual desde noviembre hasta mayo en cada tratamiento, en el que se analizaron $\mathrm{CE}, \mathrm{pH}$, bicarbonatos y macronutrientes, expresados en $\mathrm{mS} / \mathrm{cm}$ y $\mathrm{mmol} / \mathrm{l}$. Para determinar el contenido de fertilizante aportado se analizó la solución fertilizante real (SFR) que aportaba el sistema de riego. Se midieron los siguientes perfiles en suelo en cada tratamiento: Para determinar lo que la planta tenía a su disposición se analizó la solución del suelo (SS) a $15 \mathrm{~cm}$ de profundidad usando sondas de succión. Y para determinar lo que la planta no había tomado se analizó la solución de drenaje o lixiviado (SD) obtenidas de lisímetros de drenaje.

Para la recogida de la solución de entrada o gotero se instaló en cada tratamiento una cinta en paralelo para no afectar al cultivo. Para la recogida de la solución de suelo se instalaron sondas de succión en cada tratamiento, con capsula cerámica francesa de $63 \mathrm{~mm}$ diámetro, a $15 \mathrm{~cm}$ de profundidad, y ambas a $10 \mathrm{~cm}$ de la cinta de riego. Para la recogida de la solución de drenaje se utilizaron los lisímetros de drenaje instalados en cada tratamiento (Gavilán y col., 2014). Por último, para la recogida de muestra vegetal se tomaron hojas adultas más jóvenes, completamente desarrolladas, con peciolos, en un número de 100 representativas de cada tratamiento.

Con estos datos analíticos se analizaron los cuatro tratamientos, determinando el fertilizante aportado y lo que la planta no ha tomado, expresados en $\mathrm{mS} \mathrm{cm}^{-1} \mathrm{y} \mathrm{mmol} \mathrm{I}^{-1}$, usando las relaciones SS/SFR y SD/SFR de cada ion. Se estimó la acumulación de $\mathrm{Cl}^{-}$y de $\mathrm{Na}^{+}$, y la reconcentración de $\mathrm{NO}_{3}{ }^{-}$respecto al $\mathrm{Cl}^{-}$y K/Na. Para relacionar la eficiencia en el uso del abonado con la eficiencia del agua de riego se convirtieron los nutrientes analizados de $\mathrm{mmol} / \mathrm{l}$ a Unidades Fertilizantes $\mathrm{mgr} \mathrm{I}^{-1}$, y se comparó entrada respecto a salida de nutrientes y su aprovechamiento por la planta (\% de cada volumen de solución). Por último, se recogieron muestras vegetales tomando hojas adultas representativas de cada tratamiento. Se analizaron todos los macro (Nitrógeno \%, Fósforo \%, Potasio \%, Calcio \%, Magnesio \%) y microelementos (Hierro ppm, Zinc ppm, Manganeso ppm, Cobre ppm, Boro ppm, Sodio ppm). Se analizó si existían diferencias de concentración de nutrientes en hojas entre tratamientos, la evolución y curvas de estos durante el ciclo del cultivo.

\section{3- Resultados}

\subsection{Evolución de los fertilizantes aplicados en cada tratamiento (SFR)}

El promedio de salinidad (CE) de las muestras analizadas durante la campaña, en la SFR o entrada al cultivo, fue de $0,4 \mathrm{mS} / \mathrm{cm}$, comenzando con valores mínimos de 0,25 y llegando hasta $0,7 \mathrm{mS} / \mathrm{cm}$ al final del cultivo. (Figura 1). 


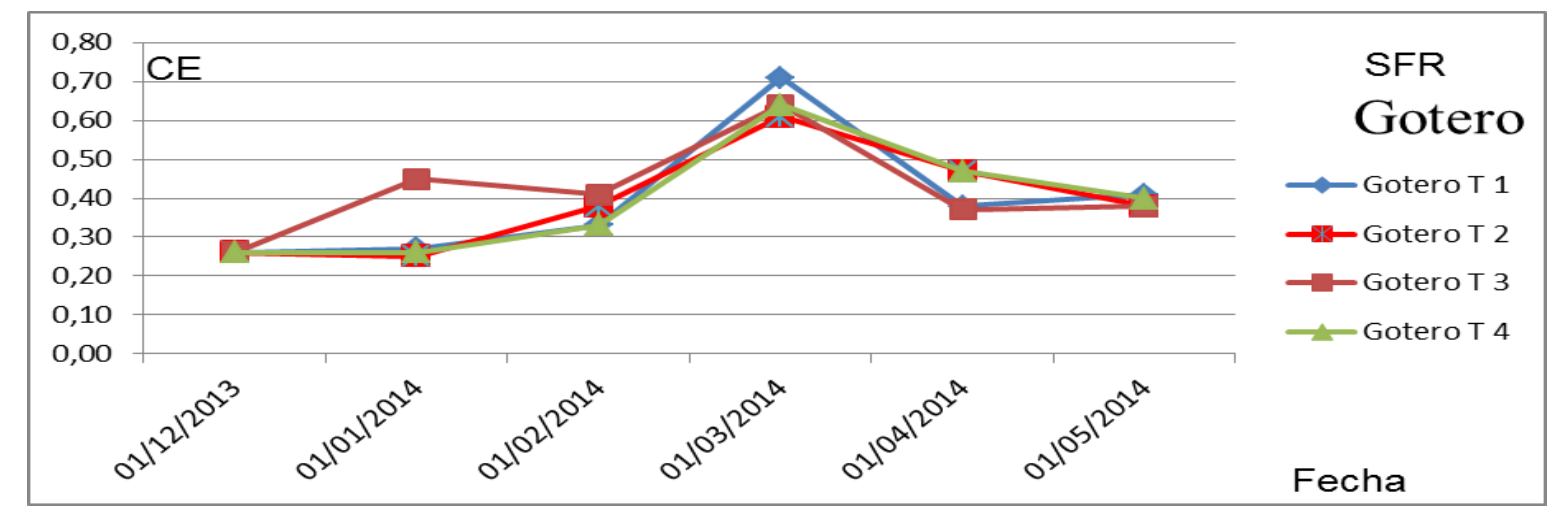

Figura 1. Evolución de los valores de CE de la SFR de entrada al cultivo en campaña.

\subsection{Comparación de las relaciones solución de nutrientes de Salida/Entrada al cultivo en cada tratamientos (SS/SFR, SD/SFR)}

Para comparar la solución nutritiva que entraba al sistema suelo-planta con la que quedaba en el suelo y con lo que le sobraba en el drenaje, en cada tratamiento se analizaron las relaciones SD/SFR, SS/SFR, y se compararon los valores promedios de los análisis de toda la campaña.

En la solución de suelo (SS) los promedios de CE de las muestras, de la relación SS/SFR, fueron de $3,52 \mathrm{mS} / \mathrm{cm}$ (T1), 1,51 (T2), 2,52 (T3), y 4,71 mS/cm (T4), con una desviación típica media de 1,37. (Tabla 1). Presentó menor concentración de nutrientes en la solución del suelo (SS) el tratamiento que recibió menor volumen de agua de riego y con caudal de cinta intermedio T2 $\left(3.8 \mathrm{I} \mathrm{h}^{-1} \mathrm{~m}^{-1}\right)$, y presentó mayor concentración de nutrientes y mayor valor de esta relación de CE el tratamiento con mayor volumen de riego $\mathrm{T} 4$, concretamente en $\mathrm{NO}_{3}^{-}, \mathrm{Cl}-$ y $\mathrm{Ca}++$ y $\mathrm{Mg}^{++}$.

\begin{tabular}{|c|c|c|c|c|c|c|c|c|c|}
\hline SS/SFR & $\mathrm{CE}$ & $\mathrm{NO}_{3}{ }^{-}$ & $\mathrm{PO}_{4} \mathrm{H}_{2}-$ & $\mathrm{SO}_{4}$ & $\mathrm{Cl}^{-}$ & $\mathrm{Na}^{+}$ & $\mathrm{K}^{+}$ & $\mathrm{Ca}^{++}$ & $\mathrm{Mg}^{++}$ \\
\hline Sondas T1 & 3,52 & 2,43 & 1,49 & 5,00 & 5,69 & 1,17 & 1,62 & 15,43 & 5,98 \\
\hline Sondas T2 & 1,51 & 2,21 & 0,60 & 1,54 & 1,90 & 1,34 & 1,39 & 3,12 & 4,59 \\
\hline Sondas T3 & 2,52 & 2,24 & 0,55 & 8,95 & 1,54 & 1,12 & 0,74 & 3,48 & 4,93 \\
\hline Sondas T4 & 4,71 & 3,62 & 0,94 & 1,73 & 11,53 & 1,00 & 1,43 & 30,34 & 21,45 \\
\hline Desviación & 1,37 & 0,67 & 0,44 & 3,48 & 4,64 & 0,14 & 0,38 & 12,84 & 8,16 \\
\hline
\end{tabular}

Tabla 1. Comparación de valores promedios de las relaciones SS/SFR. (CE $\mathrm{mS} / \mathrm{cm}$, iones $\left.\mathrm{mmol} \mathrm{I}^{-1}\right)$

En drenaje en la relación SD/SFR el promedio fue de 1,19 (T1), 1,16 (T2), 0,93 (T3), y 1,14 (T4), con diferencias muy pequeñas entre tratamientos, con una desviación típica media de 0,12. (Tabla 2). Presentaron menor valor en esta relación de CE y mayor aprovechamiento de nutrientes el tratamiento con menor volumen de agua y caudal de cinta de riego de $5 \mathrm{I} \mathrm{h}^{-1} \mathrm{~m}^{-1}$, el T3. Presentó mayor valor de esta relación y mayor perdida por drenaje el resto de tratamientos, con valores similares el T1 $(1,19)$, T2 $(1,16)$, y T4 $(1,14)$. El tratamiento con mayor volumen de agua $\mathrm{T} 4$ presento mayor drenaje en $\mathrm{NO}_{3}^{-}, \mathrm{PO}_{4} \mathrm{H}_{2}^{-} \mathrm{y} \mathrm{K}^{+}$.

\begin{tabular}{|c|c|c|c|c|c|c|c|c|c|}
\hline SD/SFR & $\mathrm{CE}$ & $\mathrm{NO}_{3}^{-}$ & $\mathrm{PO}_{4} \mathrm{H}_{2}^{-}$ & $\mathrm{SO}_{4}{ }^{-}$ & $\mathrm{Cl}^{-}$ & $\mathrm{Na}^{+}$ & $\mathrm{K}^{+}$ & $\mathrm{Ca}^{++}$ & $\mathrm{Mg}^{++}$ \\
\hline Lisimetro T1 & 1,19 & 2,26 & 0,98 & 2,28 & 1,07 & 1,18 & 1,10 & 6,60 & 1,96 \\
\hline Lisímetro T2 & 1,16 & 2,68 & 0,52 & 1,79 & 1,02 & 1,20 & 0,83 & 5,27 & 1,65 \\
\hline Lisímetro T3 & 0,93 & 2,31 & 0,24 & 2,17 & 0,89 & 1,10 & 1,07 & 4,76 & 1,11 \\
\hline Lisímetro T4 & 1,14 & 3,45 & 2,20 & 2,26 & 1,06 & 1,08 & 1,85 & 5,43 & 1,63 \\
\hline Desviación & 0,12 & 0,55 & 0,87 & 0,23 & 0,08 & 0,06 & 0,44 & 0,78 & 0,35 \\
\hline
\end{tabular}


Tabla 2. Comparación de valores promedios de las relaciones SD/SFR. (CE mS/cm, iones $\mathrm{mmol} \mathrm{I}^{-1}$ )

Los valores del \% de CE del drenaje $\left(\left(\mathrm{CE}_{\mathrm{SD}}-\mathrm{CE}_{\mathrm{SFR}}\right) / \mathrm{CE}_{\mathrm{SFR}}\right)$ * 100$)$ fueron similares entre los distintos tratamientos, siguiendo las mismas curvas durante toda la campaña, sufriendo en marzo una bajada en su evolución también en todos los tratamientos. (Figura 2). El nivel máximo de todos los tratamientos fue de $44 \%$. A pesar de la aplicación de distintos volúmenes de riego concurren para esta coincidencia entre distintos tratamientos la baja CE de la solución nutritiva de entrada al cultivo SFR.

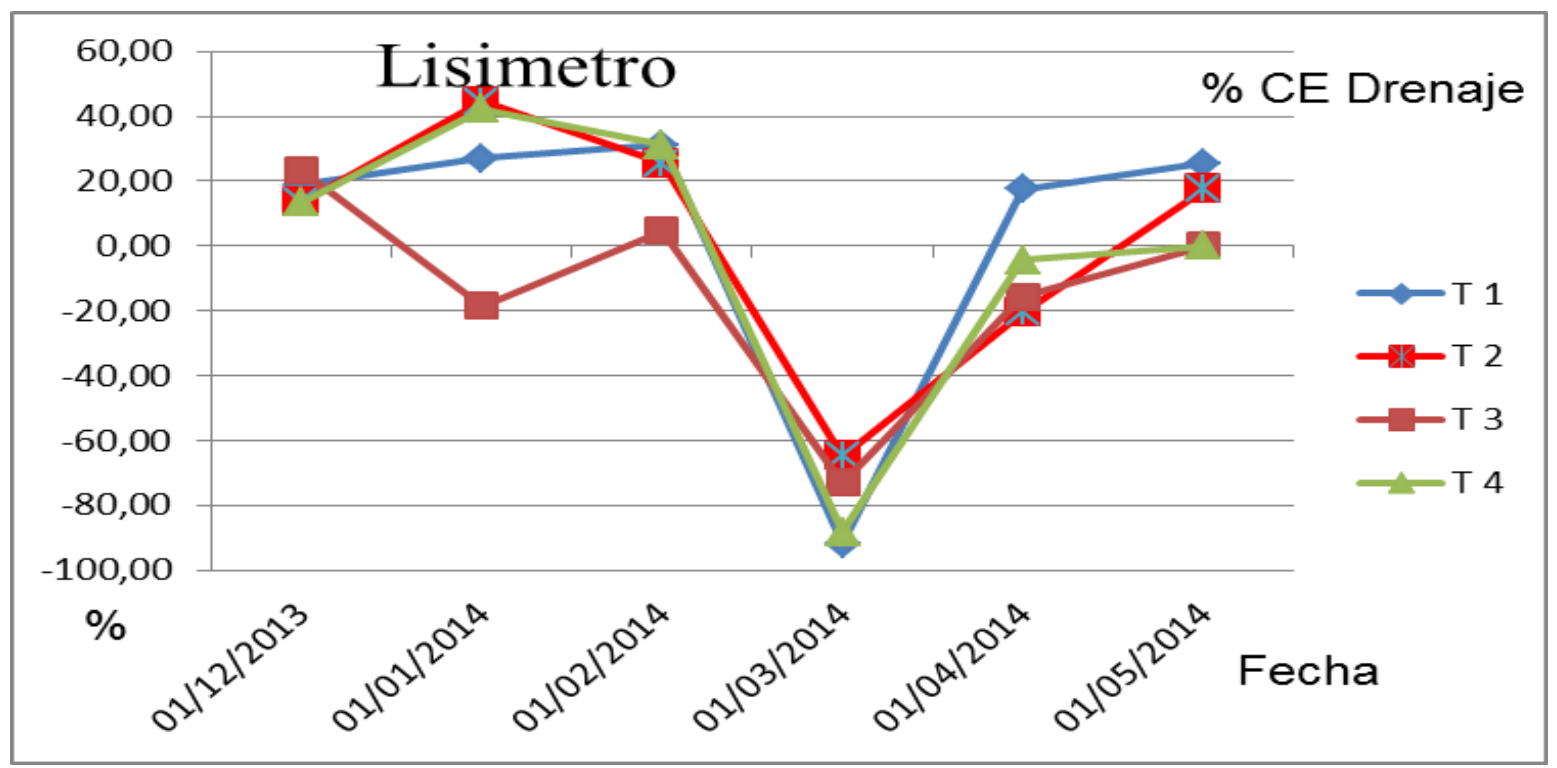

Figura 2. Los valores de \% CE de drenaje durante la campaña

\subsection{Comparación de la acumulación de cloro y de sodio}

Como indicador de que las dotaciones de agua fueron suficientes para evitar la acumulación salina, de cloro y sodio, en el medio, se aplicaron las siguientes fórmulas para el cálculo del \% de cloro que entraba al sistema respecto al que tenía en la SS y al que salía por drenaje:

\section{Para el cloro: [1-(Cl de la SFR / Cl de la SD)] x 100 \\ Para el sodio: [1-(Na de la SFR / Na de la SD)] x 100}

Para estos cálculos se debe tener en cuenta que la CE de la SFR de entrada al cultivo es baja (aproximadamente $\mathrm{CE}=0,5 \mathrm{mS} \mathrm{cm}^{-1}$ ) y los niveles de $\mathrm{Cl}$ y $\mathrm{Na}$ también lo son $\left(2,5-2 \mathrm{mmol} \mathrm{I}^{-1}\right)$.

Los promedios de reconcentración de $\mathrm{Cl}$ y $\mathrm{Na}$ dieron valores bajos durante toda la campaña en todos los tratamientos. En drenaje los valores de cloro se situaron en $5 \%$ (T1), $2 \%$ (T2), $1 \%$ (T3), y 5\% (T4) (Figura 3). En drenaje los valores de sodio se situaron en $12 \%$ (T1), 15\% (T2), 9\% (T3), y 6\% (T4) (Figura 3). No superando valores de $30 \%$ ni en Cl ni en $\mathrm{Na}$. No se produjeron acumulación de $\mathrm{Cl}^{-}$ni de $\mathrm{Na}^{+}$en ningún tratamiento. 


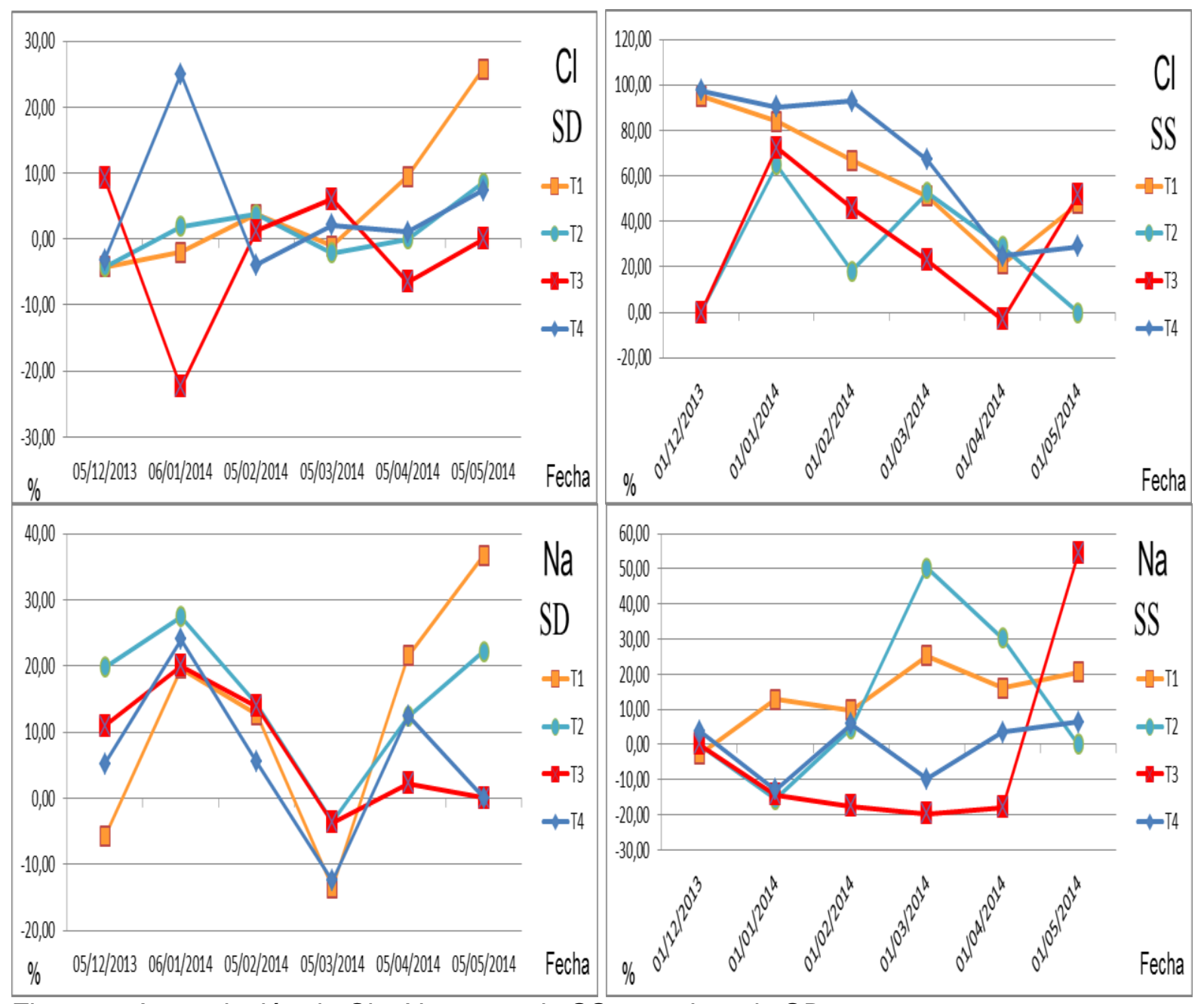

Figura 3. Acumulación de $\mathrm{Cl}$ y $\mathrm{Na}$ en suelo SS y en drenaje $\mathrm{SD}$

\subsection{Comparación de la eficiencia del abono/eficiencia riego}

Para relacionar la eficiencia en el uso del abonado con la eficiencia del agua de riego: se convirtieron los mmol $\mathrm{I}^{-1}$ analizados a Unidades Fertilizantes en $\mathrm{mg}^{-1}$, y se expresó en \%, por cada 100 litros de agua de riego que entraba cuantas unidades fertilizantes aportaban, y su correspondiente volumen de drenaje cuantas aportaba y su relación. Se comparó entrada respecto a salida de nutrientes y su aprovechamiento por la planta. (Figura 4).

Los promedios de la relación eficiencia abono/eficiencia riego de todos los macronutrientes mostraron valores mayores de eficiencia en el tratamiento con mayor eficiencia de riego y menor volumen de drenaje el T3 (88\%), seguidos de T2 (76\%), y T1 (75\%). El valor menor de esta relación lo presentó el tratamiento con menor eficiencia de agua y mayor porcentaje de drenaje el T4 (63\%). En este tratamiento este mayor volumen de drenaje no fue compensado con una menor CE de drenaje o consumo por la planta respecto a los otros tratamientos, por este motivo quedó más desfavorable en esta relación.

Estos valores fueron menores en la media de los 4 tratamientos en los nutrientes con movimientos en el suelo por flujo de masa, nitrógeno (42\%) y calcio $(72 \%)$, y fueron mayores en los nutrientes que se mueven en el suelo por difusión, fosforo (97\%) y potasio (88\%). 


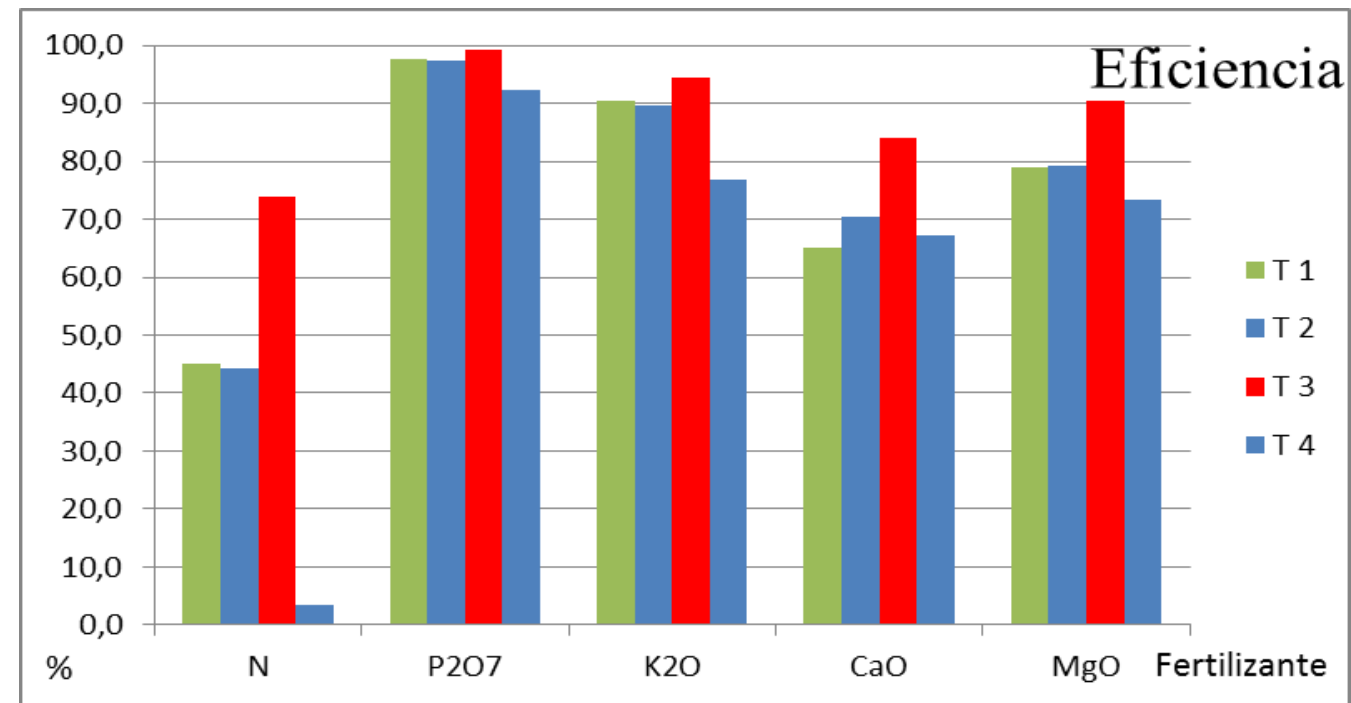

Figura 4. Relación eficiencia abono/eficiencia riego de los macronutrientes para los cuatros tratamientos.

\subsection{Comparación de los análisis foliares}

En cuanto a los análisis foliares, presentaron niveles más altos en hoja el T4 respecto al resto, en $\mathrm{N}$ (diferencia $0,23 \%$ ) y $\mathrm{K}^{+}(0,16 \%)$, no existieron diferencias de valor en $\mathrm{P}$ y $\mathrm{Mg}$ y más bajos en Ca y Microelementos. Se obtuvieron los siguientes resultados (Figura $5)$.

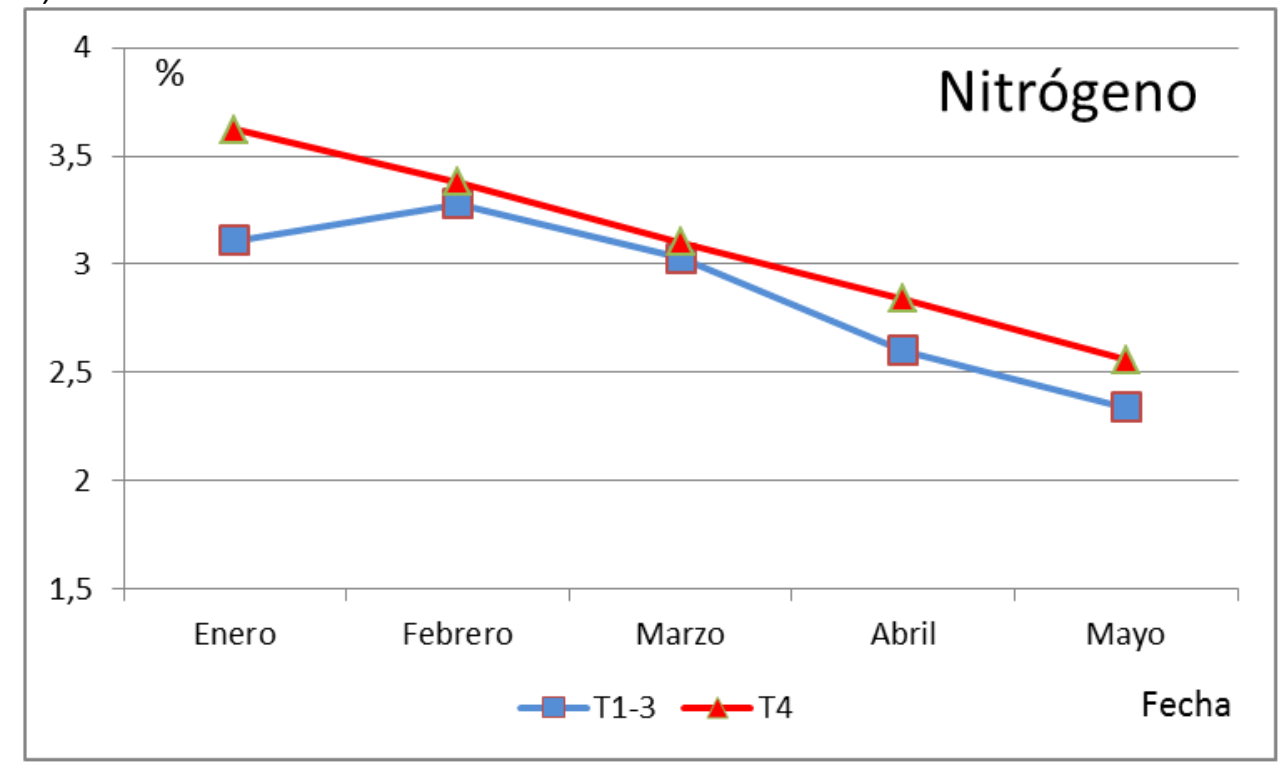


XXXIII Congreso Nacional de Riegos

Universitat Politècnica de València, Valencia 2015

DOI:http://dx.doi.org/10.4995/CNRiegos.2015.1437

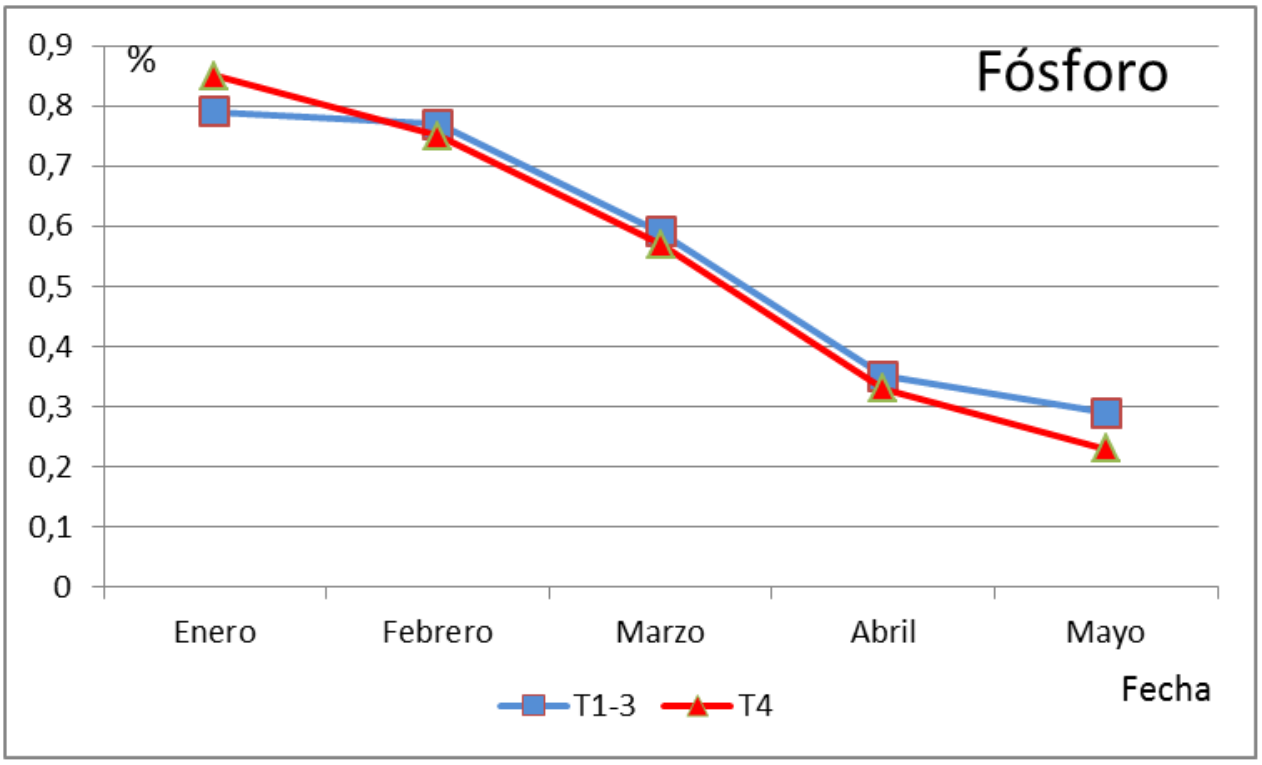


XXXIII Congreso Nacional de Riegos

Universitat Politècnica de València, Valencia 2015

DOI:http://dx.doi.org/10.4995/CNRiegos.2015.1437
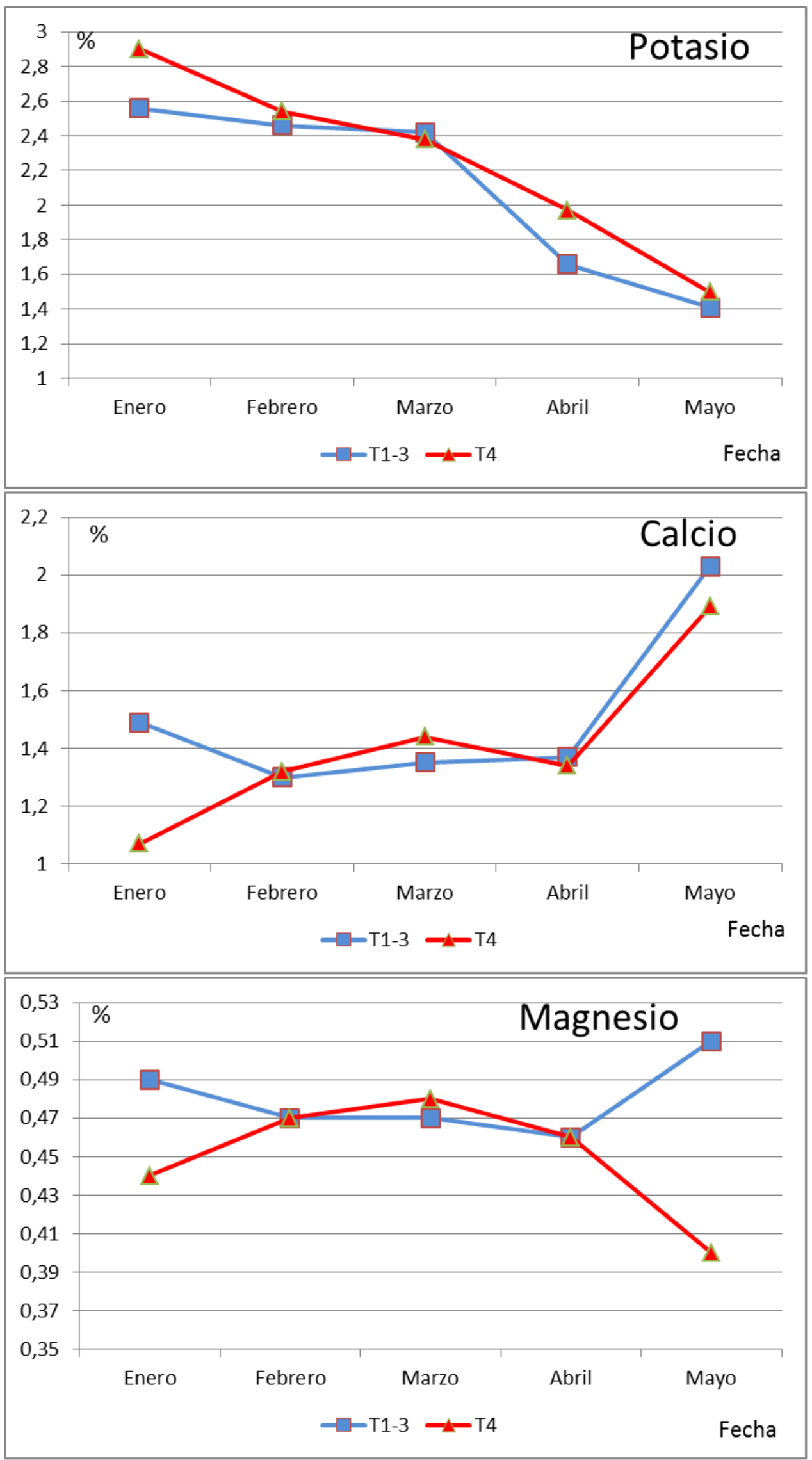

Figura 5. Niveles de macronutrientes en análisis de hoja en Sabrina. 


\section{4- Conclusiones y Recomendaciones}

Con la concentración de entrada de nutrientes al cultivo aplicado y los volúmenes de agua de riego aplicados, no se observaron grandes diferencias ni en la solución del suelo ni en la de drenaje, en los distintos tratamientos, con volúmenes de agua de riego distintos. No se produjeron acumulaciones de sales en zonas radiculares en tratamientos con distintos volúmenes de riego. Las pequeñas diferencias aparecieron en favor de los menores volúmenes de agua aplicados, sobre todo y por este orden T3 y T2. Menores volúmenes de agua aplicados presentaron mayores eficiencias y aprovechamiento en el uso de los fertilizantes. Sin embargo, en los análisis de hoja aparecieron niveles más altos sobre todo de $\mathrm{N}$ y $\mathrm{K}$ en el tratamiento con más agua y fertilizante T4.

La producción no presentó diferencias entre tratamientos, $516 \mathrm{gr} /$ plantas (T1), 544 (T2), 556 (T3), y 560 (T4).

La concentración de sales en SS y SD fue muy parecida a la del gotero, no existiendo interacción ni retención en el suelo. Se concluye que es indispensable un aporte continuo de nutrientes equilibrados para cada etapa del desarrollo de la planta, suministrados al ritmo que los pueda absorber la planta, para evitar pérdidas.

Recomendamos un siguiente estudio para conocer la concentración de sales a aplicar en cada momento del riego, teniendo en cuenta que con los riegos de precisión es más limitada la fertilización que con el riego tradicional.

\section{Agradecimientos}

Este trabajo no habría podido ser realizado sin el patrocinio de las empresas SUREXPORT SL, y GAT FERTILIQUIDOS.

\section{5- Referencias}

Duchein, M.C., Baile, M., Balle, A., (1994)."Water use efficiency and nutrient consumption of greenhouse rose grown in rockwool". Acta Horticulturae.

Gavilán, P., Lozano, D., Ruiz, N., Molina, F., 2014. El riego de la fresa en el entorno de Doñana. Evapotranspiración, coeficientes de cultivo y eficiencia del riego. XXXII Congreso Nacional de Riegos. Asociación Española de Riegos y Drenajes. Madrid, 1012 junio de 2014.

Cadahía, C. (1988). Fertilización en riego por goteo de cultivos hortícolas, Edit. ERT

Fertilizantes, Unión Explosivos Rio Tinto, Madrid. 